\title{
More severe impairment of manual dexterity in bipolar disorder compared to unipolar major depression
}

\author{
Daimei Sasayama ${ }^{\mathrm{a}, \mathrm{b} *}$, Hiroaki Hori ${ }^{\text {a }}$, Toshiya Teraishi ${ }^{\mathrm{a}}$, Kotaro Hattori ${ }^{\mathrm{a}}$, Miho Ota ${ }^{\mathrm{a}}$, \\ Junko Matsuo $^{\text {a }}$, Yumiko Kawamoto ${ }^{\text {a }}$, Yukiko Kinoshita ${ }^{\text {a }}$, Miyako Hashikura ${ }^{\text {a }}$,
} Naoji Amano ${ }^{\text {b }}$, Teruhiko Higuchi ${ }^{\text {c }}$, Hiroshi Kunugi a, d

\footnotetext{
${ }^{a}$ Department of Mental Disorder Research, National Institute of Neuroscience, National Center of Neurology and Psychiatry, 4-1-1, Ogawahigashi, Kodaira, Tokyo, 187-8502, Japan

${ }^{\mathrm{b}}$ Department of Psychiatry, Shinshu University School of Medicine

${ }^{\mathrm{c}}$ National Center of Neurology and Psychiatry, 4-1-1, Ogawahigashi, Kodaira, Tokyo, 187-8502, Japan

${ }^{\mathrm{d}}$ CREST (Core Research of Evolutional Science \& Technology), Japan Science and Technology Agency (JST) , Tokyo, 102-0075, Japan

*Correspondence to:

Daimei Sasayama

Department of Mental Disorder Research, National Institute of Neuroscience, National Center of Neurology and Psychiatry

4-1-1, Ogawahigashi, Kodaira, Tokyo, 187-8502, Japan

TEL: +81-42-341-2712, extension 5132

FAX: +81-42-346-1744
}

Email: sasayama@ shinshu-u.ac.jp 


\section{Abstract}

BACKGROUND: Mood disorders are associated with various neurocognitive deficits.

However, few studies have reported the impairment of motor dexterity in unipolar depression and bipolar disorder. In the present study, manual dexterity was compared between unipolar major depression, bipolar disorder, and healthy controls.

METHODS: Manual dexterity was assessed by the Purdue pegboard test in 98 patients with unipolar major depression, 48 euthymic or depressed patients with bipolar disorder, and 158 healthy controls, matched for age and gender.

RESULTS: Compared to healthy controls, sum of the scores of right, left, and both hands subtests $(\mathrm{R}+\mathrm{L}+\mathrm{B})$ was significantly lower in both patients with unipolar depression and bipolar disorder $(P=0.0034$ and $P<0.0001$, respectively). Furthermore, $\mathrm{R}+\mathrm{L}+\mathrm{B}$ was significantly lower in bipolar disorder compared to unipolar depression $(P$ $=0.0016)$. Lithium dose and chlorpromazine equivalent dose of antipsychotics were significantly negatively correlated with some of the subtest scores. On the other hand, depression severity did not significantly correlate with any of the subtest scores. Difference in $\mathrm{R}+\mathrm{L}+\mathrm{B}$ between unipolar depression and bipolar disorder remained statistically significant even after controlling for gender, age, lithium dose, and chlorpromazine equivalent dose $(P=0.0028)$. 
LIMITATIONS: Bipolar patients during manic episode were not included in the study.

CONCLUSIONS: Gross movement dexterity was impaired in both patients with unipolar depression and bipolar disorder. The severity of impairment was significantly greater in patients with bipolar disorder. The functional difference between unipolar and bipolar patients may suggest different pathological conditions between the two depressive disorders.

Keywords: Unipolar Depression, Bipolar Disorder, Dexterity, Lithium, Antipsychotics 


\section{Introduction}

Classifications of mood disorders are based on the polarity of episodes. However, similar depressive symptomatology between unipolar and bipolar disorders makes the differentiation difficult in depressed patients without a history of manic episode. Although several clinical characteristics such as hypersomnia and psychotic symptoms have been suggested to be helpful in distinguishing bipolar depression from unipolar depression (Forty et al., 2008; Mitchell et al., 2001), the lack of clear-cut clinical features distinguishing the two disorders has prompted researchers to seek genetic markers and endophenotypes. A few studies have found differences in personality profiles between unipolar and bipolar depression (Akiskal et al., 2006; Mendlowicz et al., 2005; Nowakowska et al., 2005; Sasayama et al., 2011). Recent evidence suggests that several neurocognitive deficits may also serve as endophenotypes of bipolar disorder (Bora et al., 2009; Langenecker et al., 2010). However, neurocognitive impairment is observed in unipolar depression as well (Han et al., 2011; Schrijvers et al., 2009), and thus, whether there are characteristic neurocognitive deficits in bipolar disorder needs to be investigated.

Impaired dexterity is one of the neurocognitive phenotypes reported in patients with bipolar disorder (Langenecker et al., 2010; Wilder-Willis et al., 2001). On the other 
hand, some studies have also reported impaired fine motor movement in unipolar depression compared to healthy controls (Pier et al., 2004b; Swann et al., 1999). However, it remains to be elucidated whether the factors affecting dexterity and the severity of the impairment are similar in unipolar and bipolar disorder. In the present study, the Purdue pegboard test (Tiffin and Asher, 1948) was used to assess the manual dexterity in unipolar depression and bipolar disorder. The influence of depression severity and antipsychotics and lithium medications on dexterity was also examined.

\section{Methods}

\subsection{Subjects}

Subjects were 98 patients with unipolar major depressive disorder (50 patients with recurrent depression), 48 euthymic or depressive patients with bipolar disorder (8 patients with bipolar I and 40 with bipolar II disorder), and 158 healthy volunteers, matched for gender and age distributions. Participants were recruited from the outpatient clinic of the National Center of Neurology and Psychiatry Hospital, Tokyo, Japan or through advertisements in local free magazines, website announcement, notices posted in the hospital, flyers, and word of mouth. Only self-reported right-handed subjects were included in the study. Consensus diagnoses by at least two research 
psychiatrists were made according to the DSM-IV criteria (American Psychiatric Association, 1994) for unipolar major depressive disorder and bipolar disorder for enrollment in the study. Healthy participants were interviewed using the Japanese version of the Mini-International Neuropsychiatric Interview (Otsubo et al., 2005; Sheehan et al., 1998) by a research psychiatrist, and only those who demonstrated no history of psychiatric illness or contact to psychiatric services were enrolled as healthy controls. Participants were excluded from both the patient and control groups if they had a prior medical history of central nervous system disease or severe head injury, or if they met DSM-IV criteria for mental retardation, substance dependence, or substance abuse. All subjects were biologically unrelated Japanese individuals who resided in the Western part of Tokyo. Written informed consent was obtained from all subjects prior to their inclusion in the study and the study was approved by the ethics committee of the National Center of Neurology and Psychiatry, Japan.

\subsection{Measures}

\subsubsection{Purdue pegboard test}

All participants were administered the Purdue pegboard test (Model 32030,

manufactured by Lafayette Instrument Company USA) for evaluation of manual 
dexterity. The pegboard contains two vertical arrays of 25 holes in which pegs are placed one hand at a time and then with both hands simultaneously under timed conditions (30 s per trial). Scores for these measures (right, left, and both hands subtests) were derived for each trial according to how many pegs were placed within the time limit. The sum of the right, left, and both hands subtest scores $(\mathrm{R}+\mathrm{L}+\mathrm{H})$ was used as the representation of gross dexterity of the fingers, hands, and arms. Fine fingertip dexterity was assessed by the assembly subtest, which involves using both hands alternately to construct assemblies consisting of a pin, a washer, a collar and another washer. This subtest requires participants to complete as many assemblies as possible within $60 \mathrm{~s}$. The total number of pieces assembled was recorded as the score of the assembly subtest.

\subsubsection{Handgrip force}

Handgrip force was measured using a digital handgrip dynamometer (T.K.K.5401; Takei Co., Tokyo, Japan) to record the muscle strength of each hand.

Participants were instructed to exert maximum grip force while standing upright, keeping their active arm stretched down vertically close to the body. The average of the two trials for each hand was defined as the maximal handgrip force. 


\subsubsection{Hamilton Depression Rating Scale}

Depressive symptoms were assessed by an experienced research psychiatrist using the Japanese version of the GRID Hamilton Depression Rating Scale, 17-item version (HDRS) (Hamilton, 1967), which has been demonstrated to show excellent inter-rater reliability (Tabuse et al., 2007).

\subsection{Statistical analyses}

Statistical differences of demographic data among groups were evaluated by the chi-squared test for categorical variables and one-way analysis of variance (ANOVA) for continuous variables. Student t-test was used for the post hoc analysis and for the comparisons of clinical variables between unipolar and bipolar patients. Due to the non-normal distribution of the Purdue pegboard scores and handgrip force, these data were compared between the three diagnostic groups using Kruskal-Wallis test, and thereafter, pairwise comparisons between two groups were done using Mann-Whitney test. Because antipsychotics and lithium, which are often prescribed for bipolar disorder, could cause tremors that may impair manual dexterity, correlations of the pegboard scores with the dose of these medications as well as with age, gender, antidepressant 
and anxiolytic prescription status, and HDRS scores were assessed using stepwise linear regression analysis (entry criteria $P<0.05$, removal criteria $P>0.2$ ). Furthermore, analysis of covariance (ANCOVA) was performed to compare $\mathrm{R}+\mathrm{L}+\mathrm{B}$ scores between diagnostic groups while controlling for gender, age, antidepressant prescription status, lithium dose, and antipsychotic dose. $\mathrm{R}+\mathrm{L}+\mathrm{B}$ scores were squared before the ANCOVA to obtain normal distribution (Shapiro-Wilk test: $P>0.1$ ). The antipsychotic dose was calculated as chlorpromazine equivalent in $\mathrm{mg} / \mathrm{day}$ according to published guidelines (American Psychiatric Association, 1997; Inagaki et al., 1999). Statistical significance was set at two-tailed $P<0.05$. Analyses were performed using the SPSS version 11.0 (SPSS Japan, Tokyo).

\section{Results}

Table 1 shows the demographic and clinical characteristics, Purdue pegboard scores, and handgrip force test results. Age distribution did not differ across the three diagnostic groups. Although the average years of education were highest in the controls, there was no significant difference between unipolar and bipolar patients. Over $35 \%$ of unipolar patients and $60 \%$ of bipolar patients were prescribed lithium and/or antipsychotics. Antidepressants and anxiolytics were also prescribed in $69 \%$ and $59 \%$ 
of unipolar patients and $54 \%$ and $63 \%$ of bipolar patients, respectively. Patients with unipolar depression and bipolar disorder did not differ significantly in age at onset or in HDRS scores. The mean score of every subtest of the Purdue Pegboard was highest in the control group and lowest in the bipolar disorder group. Post hoc pairwise comparisons with Bonferroni corrections revealed that $\mathrm{R}+\mathrm{L}+\mathrm{B}$ scores were significantly higher in control subjects compared to unipolar and bipolar disorders and were significantly lower in bipolar disorder compared to unipolar depression. Patients with bipolar disorder also scored significantly lower in assembly subtest scores compared to control subjects, although the difference with unipolar depression did not reach statistical significance. No significant difference was observed between groups in the results of the handgrip force test. Comparison between bipolar I and bipolar II disorders did not result in significant difference in the pegboard scores. However, each bipolar subtype showed significantly lower scores in $\mathrm{R}+\mathrm{L}+\mathrm{B}$ compared to healthy controls and unipolar depression (bipolar I vs controls: $P=0.0046$, bipolar II vs controls: $P<0.0001$, bipolar I vs unipolar: $P=0.045$, bipolar II vs unipolar: $P=0.0054$; Mann-Whitney test). Table 2 shows the results of the stepwise linear regression analyses with $\mathrm{R}+\mathrm{L}+\mathrm{B}$ or assembly scores as the dependent variable. Age and gender, as well as lithium and antipsychotic chlorpromazine equivalent dose, antidepressant and anxiolytic 
prescription status (i.e., $0=$ non-prescribed and $1=$ prescribed), and HDRS scores in patient groups, were included as predictor variables. Age was negatively correlated with $\mathrm{R}+\mathrm{L}+\mathrm{B}$ and assembly scores in all diagnostic groups. Lithium dose showed significant positive correlation with assembly scores in the unipolar depression group. On the other hand, antipsychotic dose was significantly negatively correlated with $\mathrm{R}+\mathrm{L}+\mathrm{B}$ and assembly scores in bipolar disorder group and with $\mathrm{R}+\mathrm{L}+\mathrm{B}$ score in unipolar depression group. Significant negative correlation between lithium dose and $R+L+B$ in patients with bipolar disorder was also observed.

Table 3 shows the results of the ANCOVA comparing square-transformed $\mathrm{R}+\mathrm{L}+\mathrm{B}$ scores between diagnostic groups. Each pairwise comparison yielded a statistically significant result.

\section{Discussion}

Comparison with healthy controls revealed that the gross movement dexterity assessed by the $\mathrm{R}+\mathrm{L}+\mathrm{B}$ score was impaired in both unipolar and bipolar disorder patients. Furthermore, the severity of impairment was significantly greater in patients with bipolar disorder compared to patients with unipolar depression. No significant difference in handgrip force across diagnostic groups suggested that poor performance 
in the pegboard test in patients groups was not due to reduced muscle strength. Antipsychotic medications had significant negative influence on the gross movement dexterity. However, the impairment of gross movement dexterity in unipolar and bipolar disorder patients remained significant even after controlling for the effects of antipsychotic and lithium medications. Fine fingertip dexterity assessed by the assembly subtest was significantly impaired in patients with bipolar disorder.

Previous studies reported fine motor dysfunction in bipolar patients even when they were euthymic (Langenecker et al., 2010; Wilder-Willis et al., 2001). Although the patients in the present study included those in depressive states, the depression severity assessed by HDRS was not significantly correlated with the outcome of the pegboard scores. Furthermore, patients with bipolar disorder showed more severely impaired dexterity compared to patients with unipolar depression, despite the similar severity of depressive symptoms. Therefore, our results also suggest that the motor dexterity in bipolar disorder patients is impaired regardless of the presence of depressive symptoms.

Some studies have also reported fine motor slowing in patients with unipolar depression (Pier et al., 2004a, b; Schrijvers et al., 2009), consistent with our results. However, studies comparing the fine motor function between unipolar and bipolar patients are scarce. Swann et al (Swann et al., 1999) examined dexterity assessed by 
continuous tapping of the right index finger in patients with unipolar depression and bipolar disorder. Their results showed that depressed patients with unipolar depression and bipolar disorder showed equally reduced tapping speed compared to healthy controls; however, bipolar disorder patients during manic state did not show significant difference compared to the controls. On the contrary, our results suggested that patients with bipolar disorder showed more severe impairment of motor dexterity compared to patients with unipolar depression irrespective of the severity of the depressive symptoms. The different results in the study by Swann et al (Swann et al., 1999) may be due to the sample selection and the method of evaluating motor function. Participants of the study by Swann et al were inpatients while our study included only outpatients with relatively low HDRS scores. Thus, more severe depressive symptoms may have influenced the dexterity test outcomes. Also, the use of Purdue pegboard allowed us to evaluate the gross movement dexterity of fingers, hands, and arms instead of the fine motor speed of a finger assessed by the finger tapping test.

The most interesting finding of the present study was that patients with bipolar disorder were more severely impaired in motor dexterity compared to unipolar patients with similar severity of depressive symptoms. Both bipolar I and bipolar II patients, despite the small number of patients with each subtype, showed significantly lower 
scores in $\mathrm{R}+\mathrm{L}+\mathrm{H}$ compared to unipolar depression. Although bipolar patients were more likely to be prescribed with antipsychotics and/or lithium, the difference between unipolar and bipolar depression remained statistically significant even when these medications were controlled for.

The functional difference strongly suggests different pathological conditions between the two disorders. Swann et al (Swann et al., 1999) reported that the relationship between psychomotor impairment and catecholamine function may be stronger in bipolar depression than in unipolar depression. Thus, the severer impairment of dexterity observed in bipolar depression may be etiologically different from that of the unipolar depression. There are other possibilities that could explain the difference in impaired dexterity between unipolar and bipolar depression. First, some of the patients with unipolar depression in this study may go on to experience a manic/hypomanic episode and be rediagnosed as bipolar disorder. Such patients may have been the cause of decreased $\mathrm{R}+\mathrm{L}+\mathrm{H}$ scores in the unipolar depression group. Secondly, unipolar depression may lie on a continuum with bipolar disorder (Akiskal and Benazzi, 2006), and thus, may show slightly impaired dexterity compared to healthy controls. Future studies should assess the motor dexterity in bipolar spectrum conditions (Akiskal et al., 2000) to examine these possibilities. 
Another finding from the study worth noting is that antipsychotic medication had significantly negative influence on motor dexterity, which was consistent with findings in a recent study of schizophrenic subjects (Sponheim et al., 2010). Physicians should keep in mind that antipsychotics, often prescribed for those with bipolar disorder as well as unipolar depression, may enhance the disability caused by the impairment of dexterity.

There are several limitations to this study. First, the cross-sectional design did not allow any definitive conclusions as to whether the impairment of the motor dexterity preceded or resulted from illness onset. Furthermore, some patients with unipolar depression in this study may be rediagnosed as bipolar disorder in the future, and thus follow-ups are necessary for accurate diagnosis. Secondly, the number of patients with bipolar disorder was small. Larger studies are needed to compare bipolar I and II disorders. Thirdly, as the patients were limited to those receiving outpatient treatments, our subjects might have been overrepresented by milder forms of illness. Moreover, we did not include bipolar patients during the manic episode. Further studies are necessary to determine whether dexterity is dependent on the phase of the disorder. Fourthly, the self-reported handedness was not verified using a validated hand preference questionnaire. A previous study has shown that non-right handedness is associated with 
soft bipolarity in mood disorders (Fasmer et al., 2008). Therefore, different rates of mixed-handed persons in each diagnostic group may have confounded the results of the pegboard test. However, since significant impairment of dexterity in bipolar disorder was observed in both the right hand and the left hand subtests, our conclusion that dexterity is impaired in bipolar disorder is not weakened by the possible inaccuracy of the handedness. Finally, the effects of medication could not be fully controlled due to the variability in types and doses. However, analyses examining the influence of antipsychotics and lithium on the outcome of the Purdue pegboard test indicated that these medications were not the only explanatory variable to the impaired dexterity.

In conclusion, we assessed manual motor dexterity in patients with unipolar depression and bipolar disorder and confirmed that both unipolar and bipolar patients were impaired in gross motor dexterity when compared to healthy controls. However, the severity of impairment was significantly greater in bipolar disorder compared to unipolar depression, despite the similar severity of depressive symptoms. The functional difference between unipolar and bipolar depression may suggest different pathological conditions between the two depressive disorders. 


\section{References}

Akiskal, H.S., Benazzi, F., 2006. The DSM-IV and ICD-10 categories of recurrent [major] depressive and bipolar II disorders: evidence that they lie on a dimensional spectrum. Journal of affective disorders 92, 45-54.

Akiskal, H.S., Bourgeois, M.L., Angst, J., Post, R., Moller, H., Hirschfeld, R., 2000.

Re-evaluating the prevalence of and diagnostic composition within the broad clinical spectrum of bipolar disorders. Journal of affective disorders 59 Suppl 1, S5-S30.

Akiskal, H.S., Kilzieh, N., Maser, J.D., Clayton, P.J., Schettler, P.J., Traci Shea, M., Endicott, J., Scheftner, W., Hirschfeld, R.M., Keller, M.B., 2006. The distinct temperament profiles of bipolar I, bipolar II and unipolar patients. Journal of affective disorders 92, 19-33. American Psychiatric Association, 1994. DSM-IV: Diagnostic and Statistical Manual of Mental Disorders. 4th edition., Washington D.C.

American Psychiatric Association, 1997. Practice Guidelines for the Treatment of Patients with Schizophrenia. American Psychiatric Press, Washington D.C.

Bora, E., Yucel, M., Pantelis, C., 2009. Cognitive endophenotypes of bipolar disorder: a meta-analysis of neuropsychological deficits in euthymic patients and their first-degree relatives. Journal of affective disorders 113, 1-20.

Fasmer, O.B., Akiskal, H.S., Hugdahl, K., Oedegaard, K.J., 2008. Non-right-handedness is associated with migraine and soft bipolarity in patients with mood disorders. Journal of affective disorders 108, 217-224.

Forty, L., Smith, D., Jones, L., Jones, I., Caesar, S., Cooper, C., Fraser, C., Gordon-Smith, K., Hyde, S., Farmer, A., McGuffin, P., Craddock, N., 2008. Clinical differences between bipolar and unipolar depression. Br J Psychiatry 192, 388-389.

Hamilton, M., 1967. Development of a rating scale for primary depressive illness. The British journal of social and clinical psychology 6, 278-296.

Han, G., Klimes-Dougan, B., Jepsen, S., Ballard, K., Nelson, M., Houri, A., Kumra, S., Cullen, K., 2011. Selective neurocognitive impairments in adolescents with major depressive disorder. Journal of adolescence.

Inagaki, A., Inada, T., Fujii, Y., Yagi, G. (Eds.), 1999. Equivalent Dose of Psychotropics. Seiwa Shoten, Tokyo.

Langenecker, S.A., Saunders, E.F., Kade, A.M., Ransom, M.T., McInnis, M.G., 2010.

Intermediate: cognitive phenotypes in bipolar disorder. Journal of affective disorders 122 , 285-293.

Mendlowicz, M.V., Akiskal, H.S., Kelsoe, J.R., Rapaport, M.H., Jean-Louis, G., Gillin, J.C., 2005. Temperament in the clinical differentiation of depressed bipolar and unipolar major 
depressive patients. Journal of affective disorders 84, 219-223.

Mitchell, P.B., Wilhelm, K., Parker, G., Austin, M.P., Rutgers, P., Malhi, G.S., 2001. The clinical features of bipolar depression: a comparison with matched major depressive disorder patients. The Journal of clinical psychiatry 62, 212-216; quiz 217.

Nowakowska, C., Strong, C.M., Santosa, C.M., Wang, P.W., Ketter, T.A., 2005.

Temperamental commonalities and differences in euthymic mood disorder patients, creative controls, and healthy controls. Journal of affective disorders 85, 207-215.

Otsubo, T., Tanaka, K., Koda, R., Shinoda, J., Sano, N., Tanaka, S., Aoyama, H., Mimura, M., Kamijima, K., 2005. Reliability and validity of Japanese version of the Mini-International Neuropsychiatric Interview. Psychiatry and clinical neurosciences 59, 517-526.

Pier, M.P., Hulstijn, W., Sabbe, B.G., 2004a. No psychomotor slowing in fine motor tasks in dysthymia. Journal of affective disorders 83, 109-120.

Pier, M.P., Hulstijn, W., Sabbe, B.G., 2004b. Psychomotor retardation in elderly depressed patients. Journal of affective disorders 81, 73-77.

Sasayama, D., Hori, H., Teraishi, T., Hattori, K., Ota, M., Matsuo, J., Kawamoto, Y., Kinoshita, Y., Hashikura, M., Koga, N., Okamoto, N., Sakamoto, K., Higuchi, T., Amano, N., Kunugi, H., 2011. Difference in Temperament and Character Inventory scores between depressed patients with bipolar II and unipolar major depressive disorders. Journal of affective disorders 132, 319-324.

Schrijvers, D., Van Den Eede, F., Maas, Y., Cosyns, P., Hulstijn, W., Sabbe, B.G., 2009. Psychomotor functioning in chronic fatigue syndrome and major depressive disorder: a comparative study. Journal of affective disorders 115, 46-53.

Sheehan, D.V., Lecrubier, Y., Sheehan, K.H., Amorim, P., Janavs, J., Weiller, E., Hergueta, T., Baker, R., Dunbar, G.C., 1998. The Mini-International Neuropsychiatric Interview (M.I.N.I.): the development and validation of a structured diagnostic psychiatric interview for DSM-IV and ICD-10. The Journal of clinical psychiatry 59 Suppl 20, 22-33; quiz 34-57. Sponheim, S.R., Jung, R.E., Seidman, L.J., Mesholam-Gately, R.I., Manoach, D.S., O'Leary, D.S., Ho, B.C., Andreasen, N.C., Lauriello, J., Schulz, S.C., 2010. Cognitive deficits in recent-onset and chronic schizophrenia. Journal of psychiatric research 44, 421-428. Swann, A.C., Katz, M.M., Bowden, C.L., Berman, N.G., Stokes, P.E., 1999. Psychomotor performance and monoamine function in bipolar and unipolar affective disorders. Biological psychiatry 45, 979-988.

Tabuse, H., Kalali, A., Azuma, H., Ozaki, N., Iwata, N., Naitoh, H., Higuchi, T., Kanba, S., Shioe, K., Akechi, T., Furukawa, T.A., 2007. The new GRID Hamilton Rating Scale for Depression demonstrates excellent inter-rater reliability for inexperienced and experienced raters before and after training. Psychiatry research 153, 61-67. 
Tiffin, J., Asher, E.J., 1948. The Purdue pegboard; norms and studies of reliability and validity. The Journal of applied psychology 32, 234-247.

Wilder-Willis, K.E., Sax, K.W., Rosenberg, H.L., Fleck, D.E., Shear, P.K., Strakowski, S.M., 2001. Persistent attentional dysfunction in remitted bipolar disorder. Bipolar disorders 3 , $58-62$. 
Table 1: Clinical characteristics and Purdue pegboard and handgrip force test results

\begin{tabular}{|c|c|c|c|c|c|c|c|}
\hline & \multirow{3}{*}{$\begin{array}{l}\text { Healthy controls } \\
\qquad(\mathrm{N}=158)\end{array}$} & \multirow{3}{*}{$\begin{array}{c}\text { Unipolar depression } \\
\qquad(\mathrm{N}=98) \\
\end{array}$} & \multirow{3}{*}{$\begin{array}{c}\text { Bipolar disorder } \\
\qquad(\mathrm{N}=48)\end{array}$} & \multirow{3}{*}{ Statistical difference } & \multicolumn{3}{|c|}{ Post hoc pairwise comparisons } \\
\hline & & & & & \multirow{2}{*}{$\begin{array}{l}\text { Unipolar depression } \\
\text { vs controls }\end{array}$} & \multirow{2}{*}{$\begin{array}{l}\text { Bipolar disorder } \\
\text { vs controls }\end{array}$} & \multirow{2}{*}{$\begin{array}{l}\text { Unipolar depression } \\
\text { vs Bipolar disorder }\end{array}$} \\
\hline & & & & & & & \\
\hline \multicolumn{8}{|l|}{ Demographic characteristics } \\
\hline Gender (Male/Female) & $79 / 79$ & $49 / 49$ & $24 / 24$ & $\chi^{2}=0.00, P=1.00$ & & & \\
\hline Average age (years) & $44.6(14.8)$ & $44.4(13.5)$ & $44.5(14.5)$ & $\mathrm{F}=0.004, \mathrm{P}=1.00$ & & & \\
\hline Education years & $15.4(2.4)$ & $14.4(2.5)$ & $14.6(2.8)$ & $\mathrm{F}=5.36, \mathrm{P}=0.0052$ & $\mathrm{t}=3.17, \mathrm{P}=0.0017$ & $\mathrm{t}=1.94, \mathrm{P}=0.054$ & $\mathrm{t}=0.98, \mathrm{P}=0.67$ \\
\hline Age at onset & n.a. & $35.0(13.1)$ & $31.7(13.3)$ & $\mathrm{t}=1.39, \mathrm{P}=0.17$ & & & \\
\hline HDRS-17 & n.a. & $10.9(7.0)$ & $11.5(7.0)$ & $\mathrm{t}=0.502, \mathrm{P}=0.62$ & & & \\
\hline \multicolumn{8}{|l|}{ Medication status } \\
\hline Antipsychotics without Lithium (\%) & 0.0 & 25.1 & 25.0 & & & & \\
\hline Antipsychotics with Lithium (\%) & 0.0 & 6.6 & 27.1 & & & & \\
\hline Lithium without antipsychotics (\%) & 0.0 & 4.0 & 8.3 & & & & \\
\hline Other psychotropics only (\%) & 0.0 & 41.0 & 18.8 & & & & \\
\hline No psychotropic medication (\%) & 100.0 & 13.2 & 20.8 & & & & \\
\hline \multicolumn{8}{|l|}{ Purdue Pegboard } \\
\hline Right hand & $14.9(2.1)$ & $13.9(2.0)$ & $13.0(2.2)$ & $\chi^{2}=30.3, P<0.0001$ & $\mathrm{U}=\mathbf{5 7 5 1 ,} \mathrm{P}=\mathbf{0 . 0 0 0 5}$ & $\mathrm{U}=2022, \mathrm{P}<0.0001$ & $U=1719, P=0.0075$ \\
\hline Left hand & $14.1(2.0)$ & $13.3(2.1)$ & $11.9(2.6)$ & $\chi^{2}=27.8, P<0.0001$ & $\mathrm{U}=6256, \mathrm{P}=\mathbf{0 . 0 0 9 0}$ & $\mathrm{U}=1978, \mathrm{P}<0.0001$ & $U=1614, P=0.0019$ \\
\hline Both hands & $11.6(1.9)$ & $11.3(2.1)$ & $10.1(2.2)$ & $\chi^{2}=27.9, P=0.0001$ & $\mathrm{U}=7160, \mathrm{P}=0.31$ & $\mathrm{U}=2273, \mathrm{P}<0.0001$ & $U=1609, P=0.0017$ \\
\hline Right + Left + Both hands & $40.6(5.1)$ & $38.4(5.5)$ & $35.0(6.2)$ & $\chi^{2}=31.6, \mathrm{P}<0.0001$ & $\mathrm{U}=6059, \mathrm{P}=\mathbf{0 . 0 0 3 4}$ & $\mathrm{U}=1852, \mathrm{P}<0.0001$ & $U=1594, P=0.0016$ \\
\hline Assembly & $35.4(8.0)$ & $33.9(8.6)$ & $30.7(9.3)$ & $\chi^{2}=12.4, P=0.0020$ & $\mathrm{U}=6576, \mathrm{P}=0.043$ & $\mathrm{U}=2617, \mathrm{P}=\mathbf{0 . 0 0 1 1}$ & $\mathrm{U}=1889, \mathrm{P}=0.053$ \\
\hline \multicolumn{8}{|l|}{ Handgrip force test } \\
\hline Right hand & $33.1(9.2)$ & $31.9(10.7)$ & $31.2(8.4)$ & $\chi^{2}=1.95, P=0.38$ & & & \\
\hline Left hand & $31.3(8.6)$ & $29.6(10.1)$ & $29.5(8.1)$ & $\chi^{2}=2.73, P=0.26$ & & & \\
\hline
\end{tabular}

Bold indicates Bonferroni corrected significance of $\mathrm{P}<0.017$ in the post hoc analysis. 
Table 2: The results of the stepwise regression analyses

\begin{tabular}{|c|c|c|c|c|c|c|}
\hline & \multicolumn{3}{|c|}{ Right + Left + Both } & \multicolumn{3}{|c|}{ Assembly } \\
\hline & $\beta$ & $\mathrm{t}$ & $P$ value & $\beta$ & $\mathrm{t}$ & $\mathrm{P}$ value \\
\hline \multicolumn{7}{|l|}{ Healthy controls } \\
\hline Age & -0.14 & -5.81 & $<0.0001$ & -0.24 & -6.35 & $<0.0001$ \\
\hline Gender & 2.79 & 3.92 & 0.0001 & 2.71 & 2.38 & 0.018 \\
\hline \multicolumn{7}{|l|}{ Patients with unipolar depression } \\
\hline Age & -0.10 & -2.09 & 0.040 & -0.25 & -3.28 & 0.0015 \\
\hline Gender & na & na & na & na & na & na \\
\hline Lithium dose & na & na & na & 0.01 & 2.27 & 0.026 \\
\hline Antipsychotic (CP equivlent) dose & -0.01 & -2.09 & 0.039 & na & na & na \\
\hline Antidepressant medication use & na & na & na & na & na & na \\
\hline Anxiolytic medication use & na & na & na & na & na & na \\
\hline HDRS score & na & na & na & na & na & na \\
\hline \multicolumn{7}{|l|}{ Patients with bipolar dis order } \\
\hline Age & -0.19 & -3.68 & 0.0007 & -0.36 & -4.51 & $<0.0001$ \\
\hline Gender & na & na & na & na & na & na \\
\hline Lithium dose & -0.01 & -2.27 & 0.028 & na & na & na \\
\hline Antipsychotic (CP equivlent) dose & -0.02 & -3.01 & 0.0045 & -0.02 & -2.56 & 0.014 \\
\hline Antidepressant medication use & na & na & na & na & na & na \\
\hline Anxiolytic medication use & na & na & na & na & na & na \\
\hline HDRS score & na & na & na & na & na & na \\
\hline
\end{tabular}

CP: chlorpromazine; HDRS: Hamilton depression rating scale; na: not applicable (not included in the stepwise model)

Table 3: The ANCOVA pairwise comparisons of the transformed $\mathrm{R}+\mathrm{L}+\mathrm{B}$ scores of the Purdue pegboard between unipolar and bipolar patients and healthy controls

\begin{tabular}{|c|c|c|c|c|c|c|}
\hline & \multicolumn{2}{|c|}{$\begin{array}{c}\text { Unipolar depression } \\
\text { vs controls } \\
\end{array}$} & \multicolumn{2}{|c|}{$\begin{array}{c}\text { Bipolar disorder } \\
\text { vs controls } \\
\end{array}$} & \multicolumn{2}{|c|}{$\begin{array}{l}\text { Unipolar depression } \\
\text { vs Bipolar disorder }\end{array}$} \\
\hline & F value & $\mathrm{P}$ value & F value & $\mathrm{P}$ value & $\mathrm{F}$ value & $\mathrm{P}$ value \\
\hline Intercept & 257.8 & $<0.0001$ & 185.7 & $<0.0001$ & 111.2 & $<0.0001$ \\
\hline Gender & 21.0 & $<0.0001$ & 15.0 & 0.0001 & 5.1 & 0.026 \\
\hline Age & 33.2 & $<0.0001$ & 42.0 & $<0.0001$ & 9.1 & 0.0030 \\
\hline Lithium dose & 0.0 & 0.87 & 1.8 & 0.18 & 1.0 & 0.32 \\
\hline Antipsychtoic (CP equivalent) dose & 4.6 & 0.032 & 8.2 & 0.0046 & 10.1 & 0.0018 \\
\hline Diagnosis & 7.2 & $\mathbf{0 . 0 0 7 7}$ & 15.4 & 0.0001 & 9.3 & 0.0028 \\
\hline
\end{tabular}

ANCOVA was performed with the square-transformed $\mathrm{R}+\mathrm{L}+\mathrm{B}$ scores as the dependent variable, diagnosis as the independent variable, and gender, age, lithium dose, and chlorpromazine equivalent dose as covariates. Bold indicates Bonferroni corrected significance of $\mathrm{P}<0.017$.

CP: chlorpromazine; ANCOVA: analysis of covariance 